\section{Commentary: Transcatheter therapies for tricuspid regurgitation: The rise of a new standard?}

\author{
Laurent Faroux, MD, MSc, ${ }^{a}$ \\ Dimitri Kalavrouziotis, MD, FRCSC, ${ }^{\mathrm{b}}$ and \\ Siamak Mohammadi, MD, FRCSC ${ }^{\mathrm{b}}$
}

Tricuspid regurgitation (TR) represents the vast majority of tricuspid valve (TV) disease and affects more than 1.5 million patients in North America. ${ }^{1}$ The prognosis of untreated TR is generally poor, leading to frequent multiple hospital readmissions due to right heart failure, liver congestion, and kidney failure. However, isolated TV surgery remains rare and is associated with the highest perioperative mortality among all cardiac valve surgeries. ${ }^{2,3}$ Consequently, most patients with severe TR are relegated to receiving lifetime medical therapy. In this context, a recent renewed interest in lessinvasive transcatheter tricuspid valve intervention (TTVI) has emerged, leading to the development of several transcatheter devices for treating clinically significant TR.

Chung and colleagues ${ }^{4}$ provide an elegant summary of the percutaneous therapies currently in development for the treatment of TR. The authors describe the technical considerations of each device alongside the available in-human data. They report an average 30-day mortality

From the ${ }^{\mathrm{a} D i v i s i o n s}$ of Cardiology and ${ }^{\mathrm{b}}$ Cardiac Surgery, Quebec Heart and Lung Institute, Laval University, Québec City, Québec, Canada.

Disclosures: Dr Faroux received fellowship support from Institut Servier and the Association Régionale de Cardiologie de Champagne-Ardenne, as well as research grants from Biotronik, Edwards Lifesciences. and Medtronic. All other authors reported no conflicts of interest.

The Journal policy requires editors and reviewers to disclose conflicts of interest and to decline handling or reviewing manuscripts for which they may have a conflict of interest. The editors and reviewers of this article have no conflicts of interest.

Received for publication April 19, 2020; revisions received April 19, 2020; accepted for publication April 22, 2020; available ahead of print June 3, 2020.

Address for reprints: Siamak Mohammadi, MD, FRCSC, Division of Cardiac Surgery, Québec Heart and Lung Institute, 2725 Chemin Sainte-Foy, Québec City, QC, Canada G1V 4G5 (E-mail: siamak.mohammadi@fmed.ulaval.ca).

JTCVS Open 2020;2:20-1

2666-2736

Copyright (c) 2020 The Authors. Published by Elsevier Inc. on behalf of The American Association for Thoracic Surgery. This is an open access article under the CC BY-NCND license (http://creativecommons.org/licenses/by-nc-nd/4.0/).

https://doi.org/10.1016/j.xjon.2020.04.005

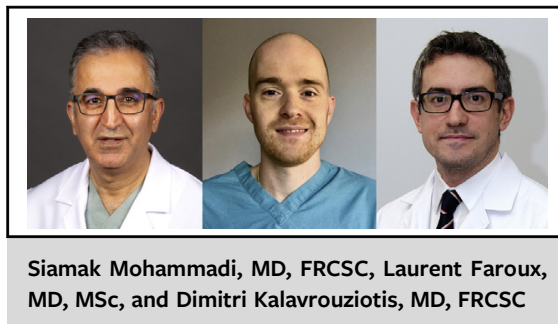

CENTRAL MESSAGE

Transcatheter options for severe

tricuspid regurgitation continue

to evolve and hold promise in

the management of this disease.

The optimal device and indica-

tions for its use remain to be

elucidated.

rate of $5 \%$ following TTVI, with encouraging results on weaker secondary end points such as the 6-minute walk test. ${ }^{5}$ However, several important challenges deserve to be highlighted, mostly linked to the anatomical features unique to the TV, which currently limit the widespread use of this type of treatment. First, unlike the aortic and mitral annuli, only the septal portion of the tricuspid annulus is fibrous. ${ }^{6}$ The combination of an incomplete fibrous annulus and the lack of calcium may increase the complexity of anchoring a device in the TV annulus. Second, electrical conduction pathways, the right coronary artery, the aortic valve, and the ostium of the coronary sinus, are important surrounding structures of the $\mathrm{TV},{ }^{5}$ which could be compromised with any radial force-induced prosthetic valve implantations or annular-based repair devices. Thus, assessing these structures with a rigorous preprocedural computed tomography protocol is of paramount importance, and an unfavorable position of the TV annulus relative to these surrounding structures can preclude successful device implantation in some cases. ${ }^{7}$ Third, the angulation in relation to the vena cava and the presence of pacemaker leads through the TV are other challenging issues. ${ }^{8}$ Finally, the proximity of the right ventricular outflow tract to the anteroseptal portion of the TV annulus could be obstructed by a long-profile device.

Consequently, TTVI is currently reserved for high-risk patients, with symptomatic functional TR before excessive dilatation of the right ventricle, ${ }^{5}$ in whom the in-hospital 
mortality rate of isolated TV surgery is estimated to be $>10 \%{ }^{2,3}$ Late referral of patients with TV disease often leads to a high prevalence of multiple comorbidities and severe right ventricle dysfunction as well as end-stage congestive heart failure signs and symptoms in many patients referred for TV surgery. This clinical reality creates an urgent need to investigate less-invasive options to treat severe TR, and few surgeons would debate that TTVI is likely to have a significant influence in the management of this morbid disease. Further studies must be performed in patients at an earlier stage of TV disease progression, and must be adequately powered to investigate hard clinical end points. Device selection must be performed based on the different pathophysiologic mechanisms leading to TV disease, and must accommodate for the increased variability of the anatomic substrate and surrounding structures compared to other cardiac valves. Only then can the relative role of TTVI compared with surgery be clearly elucidated.

\section{References}

1. Stuge O, Liddicoat J. Emerging opportunities for cardiac surgeons within structural heart disease. J Thorac Cardiovasc Surg. 2006;132:1258-61.

2. Zack CJ, Fender EA, Chandrashekar P, Reddy YNV, Bennett CE, Stulak JM, et al National trends and outcomes in isolated tricuspid valve surgery. J Am Coll Cardiol. 2017;70:2953-60

3. Alqahtani F, Berzingi CO, Aljohani S, Hijazi M, Al-Hallak A, Alkhouli M Contemporary trends in the use and outcomes of surgical treatment of tricuspid regurgitation. J Am Heart Assoc. 2017;6:e007597.

4. Chung CJ, George I. Emerging transcatheter therapies for tricuspid valve disease. J Thorac Cardiovasc Surg Open. 2020;2:14-9.

5. Asmarats L, Puri R, Latib A, Navia JL, Rodés-Cabau J. Transcatheter tricuspid valve interventions: landscape, challenges, and future directions. J Am Coll Cardiol. 2018;71:2935-56.

6. Saremi F, Sánchez-Quintana D, Mori S, Muresian H, Spicer DE, Hassani C, et al. Fibrous skeleton of the heart: anatomic overview and evaluation of pathologic conditions with CT and MR imaging. Radiographics. 2017;37:1330-51.

7. van Rosendael PJ, Kamperidis V, Kong WK, van Rosendael AR, van der Kley F, Ajmone Marsan N, et al. Computed tomography for planning transcatheter tricuspid valve therapy. Eur Heart J. 2017;38:665-74.

8. Rodés-Cabau J, Hahn RT, Latib A, Laule M, Lauten A, Maisano F, et al. Transcath eter therapies for treating tricuspid regurgitation. J Am Coll Cardiol. 2016;67: $1829-45$. 